\title{
Herding Behavior Around US Macroeconomic Announcements
}

\author{
Aymen Belgacem, IPAG Lab \& LEO-University of Orléans, France \\ Amine Lahiani, LEO-University of Orléans \& ESC Rennes School of Business, France
}

\begin{abstract}
This paper investigates the herding behavior of investors in 18 European countries around US macroeconomic announcements. By considering daily data from February 3, 2000 through July 31, 2011 and a large sample of US macroeconomic indicators, we find evidence that the intentional herding behavior intensity decreases when accounting for US macroeconomic news. The herding behavior is adopted intentionally in some European countries namely France, Switzerland and Portugal while spuriously in Greece. In addition to herding with their respective domestic markets, investors in the first three countries herd around some US macroeconomic announcements, suggesting that these investors reveal a somewhat spurious herding behavior. Findings support evidence that investors in Belgium, Finland and Ireland adopt rational investment decision making with regard to their respective domestic markets, but show pronounced herding behavior around US announcements mainly the case of Finland and Ireland.
\end{abstract}

Keywords: Stock Prices; Macroeconomic Announcements; Herding Behavior

\section{INTRODUCTION}

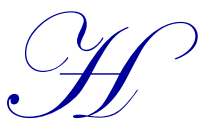

erding behavior of international financial investors is now widely argued for in many recent papers (Christie and Huang, 1995; Chang et al., 2000, Chiang et al, 2010). Understanding this pattern is extremely important for traders, risk managers, policymakers among others. Indeed, the herding behavior adopted, wittingly or not, by investors can drive the excess volatility often observed in financial markets. It can also destabilize the market and cause systemic risk (Bikhchandani and Sharma, 2001), leading to violation of the market efficiency hypothesis. Several reasons explain the existence of herding behavior among investors, mainly their psychology and desire to comply with the general consensus of the market (Devonow and Welch, 1996). Moreover, herding can be the result of following the activities of successful investors (Chiang and Zheng, 2010). This attitude leads to higher correlation among investors and hence lowers the benefits of diversification.

So far, the literature investigating the herding behavior of individual investors and institutional investors focused on two basic statistical tools namely dynamic correlation to assess the co-movement behavior (Corsetti et $a$, 2005) and Cross-Sectional Standard Deviation (CSSD) of returns (Christie and Huang, 1995). Indeed, Chiang et al (2007) separate two episodes of dynamic correlation between Asian stock returns during the Asian crisis. The first stage, started with the onset of the Asian crisis, reveals a rise in the correlation that might be due to a contagion effect and the second is characterized by continued high correlation which is due to herding behavior. Likewise, Dermirer and Kutan (2006) find no evidence of herding behavior in Chinese stock market and thus, Chinese investors are rational and use market consensus rather than mimic other investors' attitude. Chang et al. (2000) extend the work of Christie and Huang (1995) and introduce a new measure of the herding behavior based on the Cross-Sectional Absolute Deviation (CSAD). The CSAD is shown to be more powerful than the CSSD in detecting the herding behavior. The authors consider a large sample of developed and developing countries and their results are against the presence of herding behavior in the US and Hong Kong. However, results regarding South Africa and Taiwan support the presence of a nonlinear relationship between the equity returns dispersions and the underlying market price movement. More recently, Chiang et al. (2010) employed the CSAD with a slight modification of Chang et al. (2000) to examine the role of the US in explaining the herding behavior in a sample of 18 developed and developing countries. Their results show no evidence of herding behavior in Latin American countries but 
suggest evidence of a significant role of the US in driving the herding behavior in non-US markets. The herding behavior is more pronounced during rising periods in Asian markets, and during crisis periods in the US and Latin American Markets.

Moreover, some recent studies distinguish between intentional herding and spurious herding. In fact, the first arises when investors decide to mimic the decisions of other investors in the market while ignoring their own beliefs (Yao et $a l, 2013$ ). In contrast, spurious herding occurs when groups facing similar decision problems take similar decisions, considering the set of information available publicly to all investors, especially macroeconomic announcements.

Several papers have focused on Scheduled macroeconomic announcements and their impact on financial markets (Belgacem, 2013; Rigobon and Sack, 2006; Bernanke and Kuttner, 2005; Dubreuille and Mai, 2009; Dimpfel, 2011; Brenner et al., 2009). While some papers have focussed on local macroeconomic news, many recent papers have documented the impact of US announcements on foreign markets (Belgacem and Lahiani, 2012; Nikkinen and Sahlstrôm, 2004; Wongswan, 2009). Belgacem and Lahiani (2012) found a volatility transmission from U.S. to French and German stock market after the release of U.S. macroeconomic announcements. This result suggests a high correlation between global stock markets when US Macroeconomic indicators are released.

The literature on herding behavior, event studies and reactions to macroeconomic announcements has evolved independently. However, it seems to be worthwhile to relate these themes. This paper is the first attempt to measure the impact of US scheduled macroeconomic announcement on the herding behavior of individual investors by using the recent CSAD measure of the rate of increase in return dispersion. It differs from previous research at least in two aspects. First, we consider on the one hand a large sample of European countries (i.e 18 countries) including countries of Western Europe, Eastern Europe as well as Scandinavia and on the second hand and unlike previous studies (Chang et al., 2000; Dermirer and Kutan, 2006; Chiang et al., 2010) it encloses a large set of observations. Second, we investigate to what extend the scheduled US macroeconomic announcements impact the intensity of the intended herding behavior of investors. Moreover, our study nests previous works attempting to differentiate the intentional and spurious components of the mimicry attitude of investors.

The remaining of the paper is organized as follows. In section 2 we introduce the econometric methodology. Data on stock markets and US macroeconomic announcements are presented in section 3. In section 4 we present a discussion of the main results. Section 5 concludes.

\section{ECONOMETRIC METHODOLOGY}

In this section, we present the empirical methodology used to further investigate whether investors in European stock markets herd after the release of US macroeconomic announcements. To the best of our knowledge there were no previous studies that attempted to empirically answer this question. Hence, our study contributes to the related literature, by introducing a new empirical model with a large set of US macroeconomic announcements.

The main intuition behind the methodology used in this paper is that the investors' behavior is changing depending on the market conditions. In calm periods, investors are more likely to trade using their own private information, thus, the dispersion around the cross sectional market returns increase. But in periods of unusual market movements, managers ignore their own beliefs in favor of the market consensus and imitate the investment decisions of others. This attitude, when adopted by a large number of investors leads to stock returns tendency to cluster around the overall market return (Chiang and Zheng, 2010). Under this intuition of herding behavior, a natural measure of the market response is the decrease of the dispersion of returns. The literature about this question has distinguished between two main measures of dispersion. The first is the CSSD which is the average proximity of individual returns to the mean, as described in Christie et al. (1995). Formally, it is defined as follows:

$\operatorname{CSSD}=\sqrt{\frac{\sum_{i=1}^{N}\left(R_{i, t}-R_{m, t}\right)^{2}}{N-1}}$ 
where $N$ is the number of industries in the portfolio, $R_{i_{3} t}$ is the observed stock return of industry $i$ at time $t, R_{m_{s} t}$ is the cross-sectional average stock of $N$ returns in the portfolio at time t. As this measure is sensitive to outliers, Christie et al (1995) and Chiang and Zheng (2010) suggest use a more powerful measure of dispersion for capturing the influence of herding behavior namely the cross-sectional absolute deviation CSAD defined as follows:

$\operatorname{CSAD}=\frac{1}{N} \sum_{i=1}^{N}\left|R_{i, t}-R_{m_{i} t}\right|$

This measure suggests that if herding occurs, investors will make similar decisions, leading to lower return dispersions. With this measure of stock return dispersions, Chiang et al. (2010) set up the following herding equation:

$C S A D_{t}=\alpha+\beta R_{m, t}+\gamma\left|R_{m, t}\right|+\theta R_{m, t}^{2}+\varepsilon_{t}$

Chiang et al. (2010) note that under rational expectations hypothesis, a linear relationship between the return dispersion and the return of market portfolio is anticipated. However, during periods of market stress, participants are more likely to herd around indicators such as the average consensus of all market opinions, and thus, the relation between CSAD and the average market return is more likely to be nonlinear. This effect can be detected by the market squared returns $R_{m_{s} t}^{2}$. Thus, a significant negative coefficient $\theta$ in equation (3) shows that during stress periods, the relationship between $C S A D$ and $R_{m_{i} t}^{2}$ is statistically negative and nonlinear, which supports the existence of herding behavior.

In order to take into account the reaction of stock markets to the release of US macroeconomic announcements, as it is commonly documented in the literature, and especially to test whether the intensity of herding behavior is higher around such announcement days, as one expects that investors would act simultaneously in the same way, we augment equation (3) by including eleven US macroeconomic releases. Formally, specification (3) becomes:

$\operatorname{CSAD}_{t}=\alpha+\beta R_{m, t}+\gamma\left|R_{m, t}\right|+\theta R_{m, t}^{2}+\sum_{k=1}^{11} \delta_{k} D_{k} R_{m, t}^{2}+\varepsilon_{t}$

where $D_{k}$ is a dummy variable taking the value 1 on the days of $\mathrm{k}^{\text {th }}$ news announcements, and 0 otherwise. The term $\sum_{k=1}^{11} \delta_{i, k} D_{k} R_{m, t}^{2}$ in eq. (4) allows detecting the herding behavior around the release of US macroeconomic indicators. A significant and negative $\delta_{k}$ indicates that the relationship between $C S A D$ and $R_{m, t}^{2}$ is statistically negative and nonlinear under the influence of US macroeconomic indicators, which also reflects the herding behavior around such news announcements.

\section{DATA}

Prices of eighteen European market prices indices and their relative industries are collected from DataStream International. We use daily data over the period from February $3^{\text {rd }}, 2000$ to July $31^{\text {st }}, 2011$ in order to adequately capture the rapidity and intensity of the reaction of stock markets.

Regarding macroeconomic announcements, the data sample consists of the following U.S. news which have been shown to significantly affect equity prices in recent papers ${ }^{1}$ :

- $\quad$ Consumer and producer price indices (monthly) as indicators of inflation

- Unemployment rate (monthly) considered as one of the most timely indicators of the economic state

- Industrial production (monthly), gross domestic production (quarterly), leading indicator, ISM index and trade balance to proxy the state of the economic activity

${ }^{1}$ See e.g. Jones et al. (2005), Rigobon and sack (2006), Belgacem and Lahiani (2012) 
- $\quad$ Consumer confidence index (monthly) and household consumption (monthly)

- Housing starts (monthly) as a real estate indicator

Announcement days of macroeconomic indicators are collected from both Bureau of Labor Statistics (BLS) and checked for afterwards through Bloomberg.

Table 1 reports a summary of statistics of CSAD in all countries considered in this paper. The mean values of CSAD show that the countries of Scandinavia have higher mean values compared to those of other European countries. We also notice that countries hit by the crisis show similar mean values of CSAD as other European countries except Ireland which presents the highest mean value of CSAD. Romania has a high mean value of CSAD relative to all other countries. High mean value indicates possible higher market variations. Standard deviations of CSAD across countries show a similar pattern to that of mean values with Romania showing the highest standard deviation followed by Finland and then Ireland.

\section{RESULTS AND DISCUSSION}

Estimation results of equations (3) and (4) are reported in Tables (2) and (3) respectively. As noted in the previous section, a significant and negative $\theta$ indicates that the relationship between $C S A D$ and $R_{m, t}^{2}$ is statistically negative and nonlinear reflecting herding behavior. Moreover, a negative $\delta_{k}$ shows that investors in European markets mimic the others after the release of the $k^{\text {th }}$ US macroeconomic indicator.

Table 1: Descriptive Statistics of Equally Weighted Cross-Sectional Absolute Deviations $\left(C S A D_{t}\right)$

\begin{tabular}{|c|c|c|c|c|c|c|c|c|c|}
\hline & Belgium & $\begin{array}{c}\text { Czech } \\
\text { Republic }\end{array}$ & Denmark & Finland & France & Germany & Greece & Ireland & Italy \\
\hline Mean & 0,0091 & 0,0109 & 0,0136 & 0,0129 & 0,0070 & 0,0072 & 0,0094 & 0,0161 & 0,0077 \\
\hline $\begin{array}{l}\text { Standard } \\
\text { Error }\end{array}$ & 0,0054 & 0,0079 & 0,0081 & 0,0111 & 0,0044 & 0,0068 & 0,0051 & 0,0103 & 0,0048 \\
\hline Minimum & 0,0035 & 0,0024 & 0,0042 & 0,0033 & 0,0000 & 0,0022 & 0,0026 & 0,0051 & 0,0028 \\
\hline Maximum & 0,0677 & 0,1127 & 0,1049 & 0,1553 & 0,0502 & 0,1742 & 0,0426 & 0,1185 & 0,0519 \\
\hline Skewness & 2,2723 & 3,0137 & 2,1074 & 3,4002 & 1,8269 & 12,5688 & 1,1530 & 2,1806 & 2,4444 \\
\hline Kurtosis & 11,3636 & 20,3485 & 11,7134 & 23,8532 & 6,3932 & 280,6085 & 3,0876 & 9,5891 & 12,0483 \\
\hline \multirow{2}{*}{$\begin{array}{l}\text { Jarque- } \\
\text { Bera } \\
\end{array}$} & $1,89 \mathrm{E}+04$ & $5,67 \mathrm{E}+04$ & $1,95 \mathrm{E}+04$ & $7,75 \mathrm{E}+04$ & $6,83 \mathrm{E}+03$ & $1,00 \mathrm{E}+07$ & $1,87 \mathrm{E}+03$ & $1,40 \mathrm{E}+04$ & $2,13 \mathrm{E}+04$ \\
\hline & Netherlands & Norway & Poland & Portugal & Romania & Spain & Sweden & Switzerland & UK \\
\hline Mean & 0,0093 & 0,0114 & 0,0097 & 0,0109 & 0,0150 & 0,0076 & 0,0097 & 0,0077 & 0,0074 \\
\hline $\begin{array}{l}\text { Standard } \\
\text { Error }\end{array}$ & 0,0060 & 0,0064 & 0,0052 & 0,0085 & 0,0127 & 0,0049 & 0,0067 & 0,0049 & 0,0046 \\
\hline Minimum & 0,0000 & 0,0038 & 0,0033 & 0,0031 & 0,0025 & 0,0025 & 0,0027 & 0,0023 & 0,0000 \\
\hline Maximum & 0,0567 & 0,0694 & 0,0518 & 0,1382 & 0,2823 & 0,0440 & 0,0751 & 0,0453 & 0,0406 \\
\hline Skewness & 1,8918 & 1,7405 & 1,3860 & 4,4201 & 6,2141 & 1,8346 & 2,1274 & 1,7064 & 1,6140 \\
\hline Kurtosis & 6,0646 & 7,2386 & 4,9660 & 44,4476 & 91,5949 & 5,8925 & 9,3366 & 5,3828 & 4,1280 \\
\hline $\begin{array}{l}\text { Jarque- } \\
\text { Bera }\end{array}$ & $6,44 \mathrm{E}+03$ & $8,13 \mathrm{E}+03$ & $4,08 \mathrm{E}+03$ & $2,59 \mathrm{E}+05$ & $1,08 \mathrm{E}+06$ & $6,07 \mathrm{E}+03$ & $1,33 \mathrm{E}+04$ & $5,12 \mathrm{E}+03$ & $3,46 \mathrm{E}+03$ \\
\hline
\end{tabular}

Note: This table reports the basic statistics of CSAD and their stochastic properties over the period February 3, 2000 through July $31,2011$.

Results in Table (2) show a little evidence of herding behavior in European stock markets. We note a significant and negative $\theta$ only for France, Switzerland, Sweden, Greece and Portugal, suggesting evidence of herding behavior of domestic investors in these countries, while there is no support for herding in Belgium, Germany, Finland and Ireland. Moreover, insignificant herding is likely to be present in Czech Republic, Poland, 
Romania, Norway, Denmark, Italy, Spain, Netherlands and UK as their $\theta$ coefficients are negative but not significant. Results in Table (2) give mixed results about the presence/absence of herding behavior in developed and developing countries in Europe. This result is conflicting with those of Economou et al. (2011) and Demirer and Kutan (2006) who argue that herding is more likely to be encountered in emerging markets due to their particular characteristics (underdeveloped financial system and regulatory framework, dominance of relatively few institutional investors, exposure to highly volatile international capital flows, thin trading, suboptimal market microstructure mechanisms and non-sophisticated small stockholders). Among developed countries investors in Germany, Belgium and Finland seem to make rational investment decisions, while French and Swedish investors seem to leave their own beliefs and fundamentals and adopt a herding behavior. Moreover, among developing countries there is strong evidence of herding behavior in Portugal while no support for mimicry behavior in Ireland. Likewise, there is no obvious support for herding behavior in the remaining developed and developing countries of our sample.

As for the effect of US macroeconomic announcements on the herding behavior in European countries results in Table (3) show evidence of herding behavior in France, Switzerland and Portugal. This result is in line with the findings of Table (2) with two main differences. First, the herding behavior, as measured by $\theta$, is lower when accounting for the US announcements suggesting on the one hand that the herding behavior in these countries is intentional and on the second hand that, to some extent, there is a spurious herding as investors in these countries tend to adopt similar decision making around the US macroeconomic announcements, as they seem to have similar investment decisions when exposed to the same information. Indeed, investors in France, Switzerland and Portugal react to US announcements on consumer confidence, trade balance, housing and GDP. In contrast, the herding behavior recorded previously in Greece seems to be totally spurious, as it becomes insignificant when introducing the US announcements. However, investors in Greece seem to herd around the release of the US trade balance. Second, the herding behavior in Switzerland decreased significantly when accounting for the US macroeconomic announcement as the coefficient on $R_{m_{z} t}^{2}$ becomes significant at the $10 \%$ level in specification (4), while it was significant at the 5\% level in specification (3). Moreover, results in Table (3) show that investors in Belgium, Finland and Ireland continue to take rational investment decisions but herd when there are US announcement on Consumer price index (CPI), as well as ISM (Finland) and household consumption (Ireland). In addition, when accounting for US announcements, the evidence of rational investment decision making shown by German investors is no more supported as the coefficient on $R_{m_{2} t}^{2}$ becomes insignificant in specification (4) while these investors are likely to herd around US announcement about CPI. More interestingly, investors in Czech Republic tend to herd around US surprises on industrial production, consumer confidence and trade balance. Indeed, the insignificant herding behavior recorded in Czech Republic is spurious and hence investors in this country are rather rational. 
Table 2: Estimation Results of Specification (3)

\begin{tabular}{|c|c|c|c|c|c|}
\hline Countries & $\alpha$ & $\beta$ & $Y$ & $\theta$ & $\mathbf{R}^{2}$ \\
\hline Belgium & $\begin{array}{c}0,0062 * * * \\
(48,4775)\end{array}$ & $\begin{array}{c}0,0079 \\
(0,8783)\end{array}$ & $\begin{array}{c}0,3254 * * * \\
(13,9421)\end{array}$ & $\begin{array}{c}1,9688 * * * \\
(3,0760)\end{array}$ & 0,4617 \\
\hline Czech Republic & $\begin{array}{c}0,0047 * * * \\
(34,9805)\end{array}$ & $\begin{array}{c}0,0006 \\
(0,0779) \\
\end{array}$ & $\begin{array}{c}0,6348 * * * \\
(34,7660)\end{array}$ & $\begin{array}{c}-0,0358 \\
(-0,1045)\end{array}$ & 0,7296 \\
\hline Denmark & $\begin{array}{c}0,0102 * * * \\
(47,8505)\end{array}$ & $\begin{array}{c}0,0167 \\
(1,3228)\end{array}$ & $\begin{array}{c}0,4042 * * * \\
(14,0934)\end{array}$ & $\begin{array}{c}-0,0577 \\
(-0,1339)\end{array}$ & 0,2019 \\
\hline Finland & $\begin{array}{c}0,0055 * * * \\
(35,8747)\end{array}$ & $\begin{array}{c}-0,0044 \\
(-0,4923)\end{array}$ & $\begin{array}{c}0,4714 * * * \\
(30,4089)\end{array}$ & $\begin{array}{c}1,8518 * * * \\
(10,6847)\end{array}$ & 0,7157 \\
\hline France & $\begin{array}{c}0,0047 * * * \\
(39,9043)\end{array}$ & $\begin{array}{c}0,0156 * * \\
(2,2150)\end{array}$ & $\begin{array}{c}0,2612 * * * \\
(14,4166)\end{array}$ & $\begin{array}{c}-1,1291 * * * \\
(-2,9043)\end{array}$ & 0,7764 \\
\hline Germany & $\begin{array}{c}0,0047 * * * \\
(24,4407)\end{array}$ & $\begin{array}{c}0,0053 \\
(0,2028)\end{array}$ & $\begin{array}{c}0,1934 * * * \\
(5,3383)\end{array}$ & $\begin{array}{c}4,5756 * * * \\
(3,8515)\end{array}$ & 0,4177 \\
\hline Greece & $\begin{array}{c}0,0066 * * * \\
(42,8247)\end{array}$ & $\begin{array}{c}0,0148 * * \\
(2,1683)\end{array}$ & $\begin{array}{c}0,2846 * * * \\
(12,4440)\end{array}$ & $\begin{array}{l}-1,0063 * \\
(-1,8370)\end{array}$ & 0,2634 \\
\hline Ireland & $\begin{array}{c}0,0113 * * * \\
(42,4004)\end{array}$ & $\begin{array}{c}0,0152 \\
(1,0322)\end{array}$ & $\begin{array}{c}0,4353 * * * \\
(11,1960)\end{array}$ & $\begin{array}{c}2,4270 * * * \\
(2,7915)\end{array}$ & 0,3425 \\
\hline Italy & $\begin{array}{c}0,0052 * * * \\
(44,1134)\end{array}$ & $\begin{array}{c}0,0202 * * * \\
(2,4916)\end{array}$ & $\begin{array}{c}0,2862 * * * \\
(14,0137)\end{array}$ & $\begin{array}{c}-0,3623 \\
(-0,7568)\end{array}$ & 0,2926 \\
\hline Netherlands & $\begin{array}{r}0,0063 * * * \\
(43,9867) \\
\end{array}$ & $\begin{array}{c}0,0056 \\
(0,6890) \\
\end{array}$ & $\begin{array}{c}0,3352 * * * \\
(16,2114)\end{array}$ & $\begin{array}{c}-0,4774 \\
(-1,1600)\end{array}$ & 0,2809 \\
\hline Norway & $\begin{array}{c}0,0080 * * * \\
(44,9919)\end{array}$ & $\begin{array}{c}0,0142 \\
(1,6418)\end{array}$ & $\begin{array}{c}0,3347 * * * \\
(14,5582)\end{array}$ & $\begin{array}{c}-0,6365 \\
(-1,5910)\end{array}$ & 0,2767 \\
\hline Poland & $\begin{array}{c}0,0064 * * * \\
(45,9461)\end{array}$ & $\begin{array}{c}0,0149 * * \\
(2,1362)\end{array}$ & $\begin{array}{c}0,3232 * * * \\
(15,2899)\end{array}$ & $\begin{array}{c}-0,0694 \\
(-0,1154)\end{array}$ & 0,3730 \\
\hline Portugal & $\begin{array}{c}0,0072 * * * \\
(32,6310)\end{array}$ & $\begin{array}{c}0,0415 * * * \\
(2,5134)\end{array}$ & $\begin{array}{c}0,5590 * * * \\
(13,1234)\end{array}$ & $\begin{array}{c}-2,5001 * * \\
(-2,2663)\end{array}$ & 0,1811 \\
\hline Romania & $\begin{array}{c}0,0081 * * * \\
(24,3279)\end{array}$ & $\begin{array}{c}0,0125 \\
(0,7743)\end{array}$ & $\begin{array}{c}0,5734 * * * \\
(12,5411)\end{array}$ & $\begin{array}{c}-1,0260 \\
(-1,2164)\end{array}$ & 0,3498 \\
\hline Spain & $\begin{array}{c}0,0047 * * * \\
(37,1538)\end{array}$ & $\begin{array}{c}0,0193 * * * \\
(2,6504)\end{array}$ & $\begin{array}{c}0,3222 * * * \\
(17,3586)\end{array}$ & $\begin{array}{c}-0,6400 \\
(-1,4347)\end{array}$ & 0,3210 \\
\hline Sweden & $\begin{array}{c}0,0049 * * * \\
(29,7886)\end{array}$ & $\begin{array}{c}0,0008 \\
(0,0816) \\
\end{array}$ & $\begin{array}{c}0,4490 * * * \\
(16,1999)\end{array}$ & $\begin{array}{l}-1,3494^{*} \\
(-1,8541)\end{array}$ & 0,4414 \\
\hline Switzerland & $\begin{array}{c}0,0050 * * * \\
(39,9913)\end{array}$ & $\begin{array}{c}0,0256 * * * \\
(2,8684)\end{array}$ & $\begin{array}{c}0,3782 * * * \\
(16,7084)\end{array}$ & $\begin{array}{c}-1,3774 * * \\
(-2,2205)\end{array}$ & 0,3104 \\
\hline UK & $\begin{array}{c}0,0050 * * * \\
(40,3608)\end{array}$ & $\begin{array}{c}0,0123 \\
(1,5297)\end{array}$ & $\begin{array}{c}0,2956 * * * \\
(12,7452)\end{array}$ & $\begin{array}{c}-0,6526 \\
(-1,0591)\end{array}$ & 0,2704 \\
\hline
\end{tabular}

Note: $*, * *$, and $* * *$ indicate significance of coefficients at the $10 \%, 5 \%$ and $1 \%$ significance levels. $t$-statistics are in parentheses. Standard errors are corrected for autocorrelation and heteroskedasticity using the Newey-West method. 
The Journal of Applied Business Research - September/October 2013

Volume 29, Number 5

\begin{tabular}{|c|c|c|c|c|c|c|c|c|c|c|c|c|c|c|c|c|}
\hline & Constant & $R_{n-3}$ & $R_{-\infty}$ & $K_{-a}^{x}$ & UNEMP & CPI & HCONS & IP & HOUSING & GDP & CONF & PPI & LEADING & ISM & TRADE & $\mathbf{R}^{2}$ \\
\hline Belgium & $\begin{array}{c}0,0062^{* * * * *} \\
(35,609) \\
\end{array}$ & $\begin{array}{l}0,0079 \\
(0,826) \\
\end{array}$ & $\begin{array}{c}0,3254 * * * * \\
(11,738) \\
\end{array}$ & $\begin{array}{l}1,513^{* * *} \\
(2,055)\end{array}$ & $\begin{array}{l}0,5436 \\
(0,432) \\
\end{array}$ & $\begin{array}{l}0,4089 \\
(0,264) \\
\end{array}$ & $\begin{array}{c}3,0729 * * * * \\
(2,554) \\
\end{array}$ & $\begin{array}{l}-0,5366 \\
(-0,326) \\
\end{array}$ & $\begin{array}{l}-0,3029 \\
(-0,384) \\
\end{array}$ & $\begin{array}{l}0,3272 \\
(0,426) \\
\end{array}$ & $\begin{array}{c}1,3807 * * * * \\
(2,381) \\
\end{array}$ & $\begin{array}{l}2,7641 \\
(0,966) \\
\end{array}$ & $\begin{array}{c}1,3609^{* * *} \\
(2,014)\end{array}$ & $\begin{array}{l}2,0023 \\
(1,200) \\
\end{array}$ & $\begin{array}{l}-0,2724 \\
(-0,158) \\
\end{array}$ & 0,467099 \\
\hline Czech Republic & $\begin{array}{c}, 0047^{* * * * *} \\
(32,202) \\
\end{array}$ & $\begin{array}{l}-0,0050 \\
(-0,779)\end{array}$ & $\begin{array}{c}0,6305^{* * * *} \\
(39,572) \\
\end{array}$ & $\begin{array}{l}, 2658 \\
(1,205)\end{array}$ & $\begin{array}{l}, 5447 \\
(0,537) \\
\end{array}$ & $\begin{array}{l}, 4875 \\
(0,445)\end{array}$ & $\begin{array}{c}-0,9664 \\
(-0,77) \\
\end{array}$ & $\begin{array}{c}-1,599^{* * * *} \\
(-2,897)\end{array}$ & $\begin{array}{l}-0,4401 \\
(-0,346)\end{array}$ & $\begin{array}{c}, 2911^{* * * * *} \\
(2,356)\end{array}$ & $\begin{array}{c}-2,5522^{* * *} \\
(-2,040) \\
\end{array}$ & $\begin{array}{c}-0,4818 \\
(-0,6) \\
\end{array}$ & $\begin{array}{l}, 2196 \\
(0,293) \\
\end{array}$ & $\begin{array}{l}-0,5203 \\
(-0,455) \\
\end{array}$ & $\begin{array}{c}-0,986^{* * * *} \\
(-5,342) \\
\end{array}$ & 0,733447 \\
\hline Denmark & $\begin{array}{c}0,0101 * * * * \\
(33,439)\end{array}$ & $\begin{array}{l}0,0161 \\
(1,204) \\
\end{array}$ & $\begin{array}{c}, 4183^{* * * *} \\
(11,462) \\
\end{array}$ & $\begin{array}{l}-0,1417 \\
(-0,298) \\
\end{array}$ & $\begin{array}{r}-3,6527 \\
(-1,411) \\
\end{array}$ & $\begin{array}{l}-0,3409 \\
(-0,158) \\
\end{array}$ & $\begin{array}{l}4,716 * * \\
(2,093) \\
\end{array}$ & $\begin{array}{l}-2,3056 \\
(-1,168) \\
\end{array}$ & $\begin{array}{c}-0,8862 \\
(-0,66) \\
\end{array}$ & $\begin{array}{l}5,0846^{*} \\
(1,895) \\
\end{array}$ & $\begin{array}{l}-1,7884 \\
(-0,716) \\
\end{array}$ & $\begin{array}{l}-2,305^{* * *} \\
(-2,031)\end{array}$ & $\begin{array}{l}-1,3406 \\
(-1,013) \\
\end{array}$ & $\begin{array}{c}2,4708 \\
(-1,07) \\
\end{array}$ & $\begin{array}{l}0,3442 \\
(0,398) \\
\end{array}$ & 0,206232 \\
\hline Finland & $\begin{array}{c}, 0055 * * * * \\
(25,617)\end{array}$ & $\begin{array}{l}-0,0055 \\
(-0,649) \\
\end{array}$ & $\begin{array}{c}0,4638^{* * * *} \\
(20,38) \\
\end{array}$ & $\begin{array}{c}, 7992^{* * * *} \\
(9,103) \\
\end{array}$ & $\begin{array}{l}-0,0087 \\
(-0,021) \\
\end{array}$ & $\begin{array}{c}-2,007 * * * \\
(-2,028) \\
\end{array}$ & $\begin{array}{l}1,2805 \\
(0,661)\end{array}$ & $\begin{array}{l}0,9430 \\
(1,444)\end{array}$ & $\begin{array}{c}1,0124^{*} \\
(1,67)\end{array}$ & $\begin{array}{c}0,5673^{*} \\
(1,79)\end{array}$ & $\begin{array}{l}0,9462 \\
(0,572)\end{array}$ & $\begin{array}{l}-0,3613 \\
(-0,279)\end{array}$ & $\begin{array}{c}1,8836^{* * * * *} \\
(4,017)\end{array}$ & $\begin{array}{l}-1,191^{*} \\
(-1,672)\end{array}$ & $\begin{array}{l}-0,0246 \\
(-0,12)\end{array}$ & 0,720468 \\
\hline France & $\begin{array}{c}0,0047^{* * * * *} \\
(30,58)\end{array}$ & $\begin{array}{c}0,0147 * * * \\
(2,157)\end{array}$ & $\begin{array}{c}0,2450 * * * \\
(10,624)\end{array}$ & $\begin{array}{c}-1,0132^{* * *} \\
(-2,165)\end{array}$ & $\begin{array}{l}0,4045 \\
(0,352)\end{array}$ & $\begin{array}{l}0,2872 \\
(0,305)\end{array}$ & $\begin{array}{l}0,5065 \\
(0,603)\end{array}$ & $\begin{array}{l}1,0931 \\
(1,409)\end{array}$ & $\begin{array}{c}2,471 * * * * \\
(2,357)\end{array}$ & $\begin{array}{l}9,432^{*} \\
(1,839)\end{array}$ & $\begin{array}{c}-2,0878^{* * *} \\
(-2,282) \\
\end{array}$ & $\begin{array}{l}-0,5358 \\
(-0,589)\end{array}$ & $\begin{array}{c}3,7112^{*} \\
(1,76)\end{array}$ & $\begin{array}{l}-0,1447 \\
(-0,151)\end{array}$ & $\begin{array}{l}0,1418 \\
(0,236)\end{array}$ & 0,234358 \\
\hline Germany & $\begin{array}{c}, 0043 * * * * \\
(14,72)\end{array}$ & $\begin{array}{l}-0,0004 \\
(-0,024) \\
\end{array}$ & $\begin{array}{c}0,2805^{* * * *} \\
(3,799) \\
\end{array}$ & $\begin{array}{l}2,1062 \\
(0,609) \\
\end{array}$ & $\begin{array}{l}1,0375 \\
(0,424) \\
\end{array}$ & $\begin{array}{c}-5,582 * * * * \\
(-4,273) \\
\end{array}$ & $\begin{array}{l}-1,9968 \\
(-1,024) \\
\end{array}$ & $\begin{array}{l}1,0359 \\
(0,514) \\
\end{array}$ & $\begin{array}{l}, 5116 \\
(0,228) \\
\end{array}$ & $\begin{array}{c}7,3677^{*} \\
(1,78)\end{array}$ & $\begin{array}{l}2,6237 \\
(0,899) \\
\end{array}$ & $\begin{array}{l}1,7533 \\
(0,678) \\
\end{array}$ & $\begin{array}{l}-1,2456 \\
(-0,611) \\
\end{array}$ & $\begin{array}{l}-2,4281 \\
(-1,282) \\
\end{array}$ & $\begin{array}{l}1,9220 \\
(0,952) \\
\end{array}$ & 0,43343 \\
\hline Greece & $\begin{array}{c}0,0066 \text { ***** } \\
(35,73)\end{array}$ & $\begin{array}{c}0,016^{* * * * *} \\
(2,446)\end{array}$ & $\begin{array}{c}0,2818^{* * * *} \\
(11,18)\end{array}$ & $\begin{array}{l}-0,9108 \\
(-1,501) \\
\end{array}$ & $\begin{array}{l}-0,9525 \\
(-1,224) \\
\end{array}$ & $\begin{array}{l}-0,6783 \\
(-0,459)\end{array}$ & $\begin{array}{l}-0,3792 \\
(-0,37)\end{array}$ & $\begin{array}{l}0,0298 \\
(0,04)\end{array}$ & $\begin{array}{l}, 2451 \\
(1,554)\end{array}$ & $\begin{array}{l}-2,1687 \\
(-1,313) \\
\end{array}$ & $\begin{array}{l}0,1099 \\
(0,093)\end{array}$ & $\begin{array}{l}-0,0880 \\
(-0,126)\end{array}$ & $\begin{array}{l}0,0673 \\
(0,092)\end{array}$ & $\begin{array}{l}0,7343 \\
(0,957)\end{array}$ & $\begin{array}{c}-2,263 * * * \\
(-5,379)\end{array}$ & 0,270233 \\
\hline Ireland & $\begin{array}{c}0,0115 * * * * \\
(34,407)\end{array}$ & $\begin{array}{l}0,0159 \\
(1,06)\end{array}$ & $\begin{array}{c}0,3891 * * * * \\
(8,587) \\
\end{array}$ & $\begin{array}{c}3,4226 * * * * \\
(3,232) \\
\end{array}$ & $\begin{array}{l}, 7801 \\
(0,476) \\
\end{array}$ & $\begin{array}{l}-2,1239 \\
(-1,525) \\
\end{array}$ & $\begin{array}{l}-1,4932^{*} \\
(-1,786) \\
\end{array}$ & $\begin{array}{l}-1,4549 \\
(-0,665) \\
\end{array}$ & $\begin{array}{l}-1,1665 \\
(-0,806) \\
\end{array}$ & $\begin{array}{l}-0,7290 \\
(-0,146) \\
\end{array}$ & $\begin{array}{l}1,6255 \\
(0,505) \\
\end{array}$ & $\begin{array}{l}0,0386 \\
(0,021) \\
\end{array}$ & $\begin{array}{l}0,1797 \\
(0,086)\end{array}$ & $\begin{array}{l}3,9035 \\
(1,571) \\
\end{array}$ & $\begin{array}{l}1,6321 \\
(0,74)\end{array}$ & 0,347548 \\
\hline Italy & $\begin{array}{c}, 0051 * * * * \\
(35,011) \\
\end{array}$ & $\begin{array}{l}0,019^{* * *} \\
(2,321)\end{array}$ & $\begin{array}{c}0,295 * * * \\
(12,08) \\
\end{array}$ & $\begin{array}{c}-0,4014 \\
(-0,79)\end{array}$ & $\begin{array}{l}-0,3555 \\
(-0,356) \\
\end{array}$ & $\begin{array}{l}-0,7107 \\
(-0,522) \\
\end{array}$ & $\begin{array}{l}-1,4654^{*} \\
(-1,916) \\
\end{array}$ & $\begin{array}{l}0,4517 \\
(0,359) \\
\end{array}$ & $\begin{array}{l}, 1730 \\
(0,815) \\
\end{array}$ & $\begin{array}{l}-1,8025 \\
(-1,621) \\
\end{array}$ & $\begin{array}{c}-3,6653^{* * * *} \\
(-4,017) \\
\end{array}$ & $\begin{array}{c}-2,2283^{* * * * *} \\
(-2,503) \\
\end{array}$ & $\begin{array}{l}-0,1512 \\
(-0,232) \\
\end{array}$ & $\begin{array}{l}0,0444 \\
(0,052) \\
\end{array}$ & $\begin{array}{c}1,5373^{* * * *} \\
(2,76) \\
\end{array}$ & 0,302507 \\
\hline Netherlands & $\begin{array}{c}, 0063^{* * * * *} \\
(31,185) \\
\end{array}$ & $\begin{array}{l}0,0031 \\
(0,397) \\
\end{array}$ & $\begin{array}{c}0,3359 * * * * \\
(14,472) \\
\end{array}$ & $\begin{array}{l}-0,4893 \\
(-1,181) \\
\end{array}$ & $\begin{array}{l}-1,1541 \\
(-0,684) \\
\end{array}$ & $\begin{array}{l}0,2211 \\
(0,181) \\
\end{array}$ & $\begin{array}{l}-0,4944 \\
(-0,799) \\
\end{array}$ & $\begin{array}{l}0,2698 \\
(0,467) \\
\end{array}$ & $\begin{array}{l}-0,0771 \\
(-0,067) \\
\end{array}$ & $\begin{array}{l}-1,4260 \\
(-0,584) \\
\end{array}$ & $\begin{array}{l}-1,8431 \\
(-1,212) \\
\end{array}$ & $\begin{array}{l}0,8291 \\
(1,368) \\
\end{array}$ & $\begin{array}{c}, 1239 \text { *** } \\
(2,233)\end{array}$ & $\begin{array}{c}-1,4213^{* * * *} \\
(-2,521) \\
\end{array}$ & $\begin{array}{l}-0,9760 \\
(-1,348)\end{array}$ & 0,28657 \\
\hline Norway & $\begin{array}{c}0,0079 * * * * \\
(34,694)\end{array}$ & $\begin{array}{l}0,0126 \\
(1,396) \\
\end{array}$ & $\begin{array}{c}0,3385 * * * \\
(12,468) \\
\end{array}$ & $\begin{array}{l}-0,7048 \\
(-1,613) \\
\end{array}$ & $\begin{array}{l}2,0611 \\
(1,495) \\
\end{array}$ & $\begin{array}{c}-2,7428 * * * * \\
(-2,341) \\
\end{array}$ & 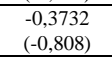 & $\begin{array}{l}-0,4572 \\
(-0,696) \\
\end{array}$ & $\begin{array}{l}3,3679^{*} \\
(1,895) \\
\end{array}$ & $\begin{array}{c}3,121^{* * * *} \\
(2,343) \\
\end{array}$ & $\begin{array}{l}-2,7140 \\
(-1,511) \\
\end{array}$ & $\begin{array}{l}0,5620 \\
(0,914) \\
\end{array}$ & $\begin{array}{l}-0,9136 \\
(-1,206) \\
\end{array}$ & $\begin{array}{l}-0,4581 \\
(-0,662) \\
\end{array}$ & $\begin{array}{c}-0,9836 * \\
(-1,875) \\
(\end{array}$ & 0,286137 \\
\hline Poland & $\begin{array}{c}0,00642^{* * * * *} \\
(36,297)\end{array}$ & $\begin{array}{c}0,0156^{* * *} \\
(1,968)\end{array}$ & $\begin{array}{c}0,3221^{* * * *} \\
(12,95)\end{array}$ & $\begin{array}{c}-0,1743 \\
(-0,22)\end{array}$ & $\begin{array}{l}0,4885 \\
(0,377)\end{array}$ & $\begin{array}{l}0,3278 \\
(0,188) \\
\end{array}$ & $\begin{array}{c}1,1915^{* * *} \\
(1,961)\end{array}$ & $\begin{array}{l}-0,6343 \\
(-0,537)\end{array}$ & $\begin{array}{l}0,3083 \\
(0,506)\end{array}$ & $\begin{array}{l}0,8761 \\
(0,423)\end{array}$ & $\begin{array}{l}-0,0170 \\
(-0,014) \\
\end{array}$ & $\begin{array}{l}1,0534 \\
(1,122)\end{array}$ & $\begin{array}{l}0,1266 \\
(0,121)\end{array}$ & $\begin{array}{l}-0,6137 \\
(-0,656) \\
\end{array}$ & $\begin{array}{l}-0,2746 \\
(-0,449) \\
\end{array}$ & 0,374049 \\
\hline Portugal & $\begin{array}{c}0,0072 * * * * \\
(30,209)\end{array}$ & $\begin{array}{c}0,0411^{\text {****** }} \\
(2,501)\end{array}$ & $\begin{array}{c}0,5550 * * * * \\
(14,041)\end{array}$ & $\begin{array}{c}-2,4344^{* * * * *} \\
(-2,74)\end{array}$ & $\begin{array}{l}-0,0619 \\
(-0,027)\end{array}$ & $\begin{array}{l}-0,0553 \\
(-0,031)\end{array}$ & $\begin{array}{l}0,9290 \\
(0,176)\end{array}$ & $\begin{array}{l}0,3916 \\
(0,246)\end{array}$ & $\begin{array}{c}-3,8856 * * \\
(-2,257)\end{array}$ & $\begin{array}{c}-9,2813 * * * * \\
(-5,959)\end{array}$ & $\begin{array}{l}-0,5080 \\
(-0,262)\end{array}$ & $\begin{array}{l}-0,6408 \\
(-0,283)\end{array}$ & $\begin{array}{l}3,1982 \\
(0,619)\end{array}$ & $\begin{array}{l}5,5399^{*} \\
(1,876)\end{array}$ & $\begin{array}{l}-1,1878 \\
(-0,965)\end{array}$ & 0,184131 \\
\hline Romania & $\begin{array}{c}, 0081^{* * * * *} \\
(18,52)\end{array}$ & $\begin{array}{l}, 0157 \\
(1,007)\end{array}$ & $\begin{array}{c}0,5540^{* * * *} \\
(9,492) \\
\end{array}$ & $\begin{array}{l}-0,7675 \\
(-0,654)\end{array}$ & $\begin{array}{c}-2,5077 * * * * \\
(-2,868) \\
\end{array}$ & $\begin{array}{l}3,1241^{*} \\
(1,652) \\
\end{array}$ & $\begin{array}{c}, 8795 * * * * \\
(2,423)\end{array}$ & $\begin{array}{l}3,0494^{*} \\
(1,732)\end{array}$ & $\begin{array}{l}-0,5372 \\
(-0,401)\end{array}$ & $\begin{array}{l}1,8887 \\
(1,286)\end{array}$ & $\begin{array}{l}-0,8383 \\
(-0,692) \\
\end{array}$ & $\begin{array}{l}-0,6135 \\
(-0,428) \\
\end{array}$ & $\begin{array}{c}-1,4358^{*} \\
(-1,781) \\
\end{array}$ & $\begin{array}{l}-1,2251 \\
(-0,669)\end{array}$ & $\begin{array}{l}-0,4344 \\
(-0,452) \\
\end{array}$ & 0,358282 \\
\hline Spain & $\begin{array}{c}0,0047^{* * * * *} \\
(26,251) \\
\end{array}$ & $\begin{array}{c}0,018^{* * * *} \\
(2,743)\end{array}$ & $\begin{array}{c}0,3199 * * * \\
(14,536) \\
\end{array}$ & $\begin{array}{l}-0,6534 \\
(-1,304) \\
\end{array}$ & $\begin{array}{l}0,6951 \\
(0,575) \\
\end{array}$ & $\begin{array}{l}0,1792 \\
(0,144) \\
\end{array}$ & $\begin{array}{l}-1,2154 \\
(-0,975) \\
\end{array}$ & $\begin{array}{l}-0,7568 \\
(-0,817) \\
\end{array}$ & $\begin{array}{c}1,7591 \\
(1,4)\end{array}$ & $\begin{array}{l}-3,5546 \\
(-1,136) \\
\end{array}$ & $\begin{array}{l}-0,3144 \\
(-0,214) \\
\end{array}$ & $\begin{array}{l}-0,5907 \\
(-0,584) \\
\end{array}$ & $\begin{array}{l}1,7735 \\
(1,418) \\
\end{array}$ & $\begin{array}{l}0,0296 \\
(0,023) \\
\end{array}$ & $\begin{array}{l}0,5591 \\
(1,437) \\
\end{array}$ & 0,323865 \\
\hline Sweden & $\begin{array}{c}0,0049 * * * * \\
(25,944)\end{array}$ & $\begin{array}{l}0,0012 \\
(0,116)\end{array}$ & $\begin{array}{c}0,4435 * * * \\
(14,079)\end{array}$ & $\begin{array}{l}-1,2510 \\
(-1,562) \\
\end{array}$ & $\begin{array}{l}0,8751 \\
(0,519)\end{array}$ & $\begin{array}{l}-0,2187 \\
(-0,202)\end{array}$ & $\begin{array}{c}-1,4573^{*} \\
(-1,68) \\
\end{array}$ & $\begin{array}{l}3,0422 \\
(1,534) \\
\end{array}$ & $\begin{array}{l}0,8427 \\
(0,716)\end{array}$ & $\begin{array}{r}2,0585 \\
(1,217)\end{array}$ & $\begin{array}{l}-0,9671 \\
(-0,636) \\
\end{array}$ & $\begin{array}{l}-1,2131 \\
(-1,454)\end{array}$ & $\begin{array}{l}0,1997 \\
(0,171)\end{array}$ & $\begin{array}{c}-2,0308^{* *} \\
(-2,16)\end{array}$ & $\begin{array}{l}1,4199 \\
(1,184)\end{array}$ & 0,446793 \\
\hline Switzerland & $\begin{array}{l}0,005 * * * * \\
(30,577) \\
\end{array}$ & $\begin{array}{c}0,019727^{* * * *} \\
(2,467)\end{array}$ & $\begin{array}{c}0,3754 * * * * \\
(14,55)\end{array}$ & $\begin{array}{c}1,2807^{*} \\
(-1,894) \\
\end{array}$ & $\begin{array}{c}-2,9943 * * \\
(-2,158)\end{array}$ & $\begin{array}{l}1,3696 \\
(1,409) \\
\end{array}$ & $\begin{array}{l}-0,9907 \\
(-1,013) \\
\end{array}$ & $\begin{array}{l}-1,0983 \\
(-1,254)\end{array}$ & $\begin{array}{c}4,8957 * * * * \\
(4,678)\end{array}$ & $\begin{array}{l}11,5608 \\
(1,574)\end{array}$ & $\begin{array}{c}-3,6634 * * * * \\
(-3,195)\end{array}$ & $\begin{array}{c}1,4076 * * * \\
(1,984)\end{array}$ & $\begin{array}{l}-0,2675 \\
(-0,413) \\
\end{array}$ & $\begin{array}{c}-0,9033 \\
(-0,7)\end{array}$ & $\begin{array}{c}-1,8183^{* * * *} \\
(-3,665)\end{array}$ & 0,322788 \\
\hline UK & $\begin{array}{l}0,005 * * * * \\
(30,823) \\
\end{array}$ & $\begin{array}{l}0,0074 \\
(1,01) \\
\end{array}$ & $\begin{array}{c}0,2865^{* * * *} \\
(11,21) \\
\end{array}$ & $\begin{array}{c}-0,2233 \\
(-0,38)\end{array}$ & $\begin{array}{l}, 8854 \\
(0,465) \\
\end{array}$ & $\begin{array}{l}-1,1930 \\
(-1,324) \\
\end{array}$ & $\begin{array}{l}-0,1595 \\
(-0,306) \\
\end{array}$ & $\begin{array}{l}0,5285 \\
(0,87) \\
\end{array}$ & $\begin{array}{l}0,0834 \\
(0,087)\end{array}$ & $\begin{array}{l}1,9562 \\
(0,515) \\
\end{array}$ & $\begin{array}{c}-3,3067 * * * * * \\
(-3,185) \\
\end{array}$ & $\begin{array}{l}-1,0503 \\
(-1,054) \\
\end{array}$ & $\begin{array}{c}1,8797 * * * \\
(2,237)\end{array}$ & $\begin{array}{l}-0,0188 \\
(-0,027) \\
\end{array}$ & $\begin{array}{c}-1,7024 * * * * \\
(-3,539) \\
\end{array}$ & 0,276842 \\
\hline
\end{tabular}

Note: ***, and $* * *$ indicate significance of coefficients at the $10 \%, 5 \%$ and $1 \%$ significance levels. $t$-statistics are in parentheses. Standard errors are corrected for autocorrelation and heteroskedasticity using the Newey-West method.

US macroeconomic announcements: UNEMP: Unemployment rate, CPI: Consumer price index, HCONS: Household consumption, , IP: Industrial production, HOUSING: Housing starts, GDP: Gross domestic product, CONF: Consumer confidence, PPI: Producer price index, LEADING: Leading indicators, ISM: ISM manufacturing, Trade: Trade balance. 
To sum up, our results show evidence of a significant impact of some US macroeconomic announcements on European stock markets. This impact is due to herding behavior around such news releases. These findings complete those of Chiang and Zheng (2010) who argue that domestic institutional investors follow those of the institutional investors in Wall Street, because of the center role of this market for processing and disseminating global investment information. Hence, since investors in major European countries consider that news released by Wall Street are valuable - and thus reliable - they significantly include them in their investment decision process. Consequently, herding behavior of European investors would be sensitive to this news.

\section{CONCLUSION}

This study examines the herding behavior of investors in European countries. Estimations are based on daily observations. This paper finds evidence to support intentional herding behavior in France, Switzerland and Portugal while spurious herding behavior was recorded in Greece. Also, German investors were found to herd around US surprises on CPI. These findings are in line with those of Belgacem and Lahiani (2012) who suspect a comovements in France and Germany in presence of US release of macroeconomic news. Investors in Belgium, Finland and Ireland are found to show rational investment decisions while depicting a herding behavior around some US macroeconomic announcements.

In particular, countries considered in this paper could be classified in three main groups. The first group includes countries in which investors tend to herd around the US indicators, in addition to their domestic markets (France, Switzerland and Portugal). The second group contains countries where investors herd around the US releases of macroeconomic news only without any herding with domestic markets (Belgium, Finland and Ireland). Countries that herd around the US macroeconomic announcements while adopt a slight insignificant herding behavior with domestic markets are in the third group.

The methodology of this paper can be extended by including the European and US financial markets to examine whether European investors herd more with the regional market rather than the international market.

\section{AUTHOR INFORMATION}

Aymen Belgacem, PhD., is an Assistant Professor of Finance at the University of Orléans and Affiliate professor at IPAG Business School. He received his PhD from the University Of Paris Ouest-la Defense. Aymen Belgacem, IPAG Lab \& LEO-University of Orléans, 6 Avenue du Parc Floral 45100 Orléans, France. E-mail: aymen.belgacem@univ-orleans.fr

Amine Lahiani, PhD., is an Associate Professor of Economics at the University of Orléans and Affiliate Professor at ESC Rennes Business School. He received his PhD from the University of Geneva. His research interests include the study of long memory models and thresholds models. Amine Lahiani, LEO-University of Orléans \& ESC Rennes School of Business, 6 Avenue du Parc Floral 45100 Orléans, France. E-mail: amine.lahiani@univorleans.fr (Corresponding author)

\section{REFERENCES}

1. Belgacem, A. and Lahiani, A., (2012). More on the Impact of US Macroeconomic Announcements: Evidence from French and German Stock Markets' Volatility. Economics Bulletin, 32, pp. 1509-1526.

2. Belgacem, A., (2013). Explaining stock market's reaction to macroeconomic announcements. Bankers, Markets and Investors n 123, pp. 43-61.

3. Bernanke, S. and Kuttner, K., (2005). What Explains the Stock Market's Reaction to Federal Reserve Policy?. Journal of Finance, 60, pp. 1221-1257.

4. Bikhchandani, S. and Sharma, S., (2001). Herd Behavior in Financial Markets. IMF Staff Papers, Palgrave Macmillan, 47, pp. 279-310.

5. Brenner M., P. Pasquariello and Subrahmanyam M., (2009). On the Volatility and Comovement of U.S. Financial Markets around Macroeconomic News Announcements. Journal of Financial and Quantitative Analysis, 44, pp. 1265-1289. 
6. Chang, E. C., Cheng, J. W. and Khorana, A., (2000). An Examination of Herd Behavior in Equity Markets: An Empirical Perpective. Journal of Banking and Finance, 24, pp. 1651-1679.

7. Chiang, T. C, Jeon, B. N. and Li, H., (2007). Dynamic Correlation Analysis of Financial Contagion: Evidenece from Asian Markets. Journal of International Money and Finance, 26, pp. 1206-1228.

8. Chiang, T. C. and Zheng, D., (2010). An Empirical Analysis of Herd Behavior in Global Stock Markets. Journal of Banking and Finance, 34, pp. 1911-1921.

9. Chiang, T. C., Li, J. and Tan, L., (2010). Empirical investigation of herding behavior in Chinese stock markets: Evidence from quantile regression analysis. Global Finance Journal, 21, pp. 111-124.

10. Christie, W. G. and Huang R. D., (1995). Following the Pied Piper: Do Individual Returns Herd Around the Market?. Financial Analysts Journal, July-August, pp. 31-37.

11. Corsetti, G., Pericoli, M. and Sbracia, M., (2005). Some Contagion, Some Interdependence: Some Pitfalls in Tests of Financial Contagion. Journal of International Money and Finance, 24, pp. 1177-1199.

12. Dermirer, R. and Kutan, A. M., (2006). Does Herding Behavior Exist in Chinese Stock Markets? Journal of International Financial Markets Institutions and Money, 16, pp. 123-142.

13. Devenow, A. and Welch, I., (1996). Rational Herding in Financial Economics. European Economic Review. 40, pp. 603-615.

14. Dimpfel, T., (2011). The Impact of US News on the German Stock Market - An Event Study Anslysis. Quarterly Review of Economics and Finance, 51, pp. 389-398.

15. Dubreuille, S. and Mai, H. M., (2009). Impact of European and American Business Cycle News on Euronext Trading. International Journal of Business, 14, pp. 124-135.

16. Economou, F., A. Kostakis and Philippas, N., (2011). Cross-country effects in herding behaviour: evidence from four south European Markets? Journal of International Financial Markets, Institutions and Money, 21, pp. 443-450.

17. Jones, B., Lin, C. T., Mansur, A. and Masih, M., (2005). Macroeconomic Announcements, volatility, and interest rate equity markets. International Review of Financial Analysis, 14, pp. 356-375.

18. Nikkinen, J. and Sahlstrôm, P., (2004). Scheduled Domestic and US Macroeconomic News and Stock Valuation in Europe. Journal of Multinational Financial Management, 14, 201-215.

19. Rigobon, R. and Sack, B., (2006). Noisy Macroeconomic Announcements, Monetary Policy, and Asset Prices. NBER Working Paper number 12420.

20. Wongswan, J., (2009). The Response of Global Equity Indexes to US Monetary Policy Announcements. Journal of International Money and Finance, 28, pp. 344-365.

21. Yao, J., Ma, C. and He, W. P., (2013). Investor Herding Behavior of Chinese Stock Market. International Review of Economics and Finance, In Press. 


\section{NOTES}

